\title{
High Levels of Serum IgG for Opisthorchis viverrini and CD44 Expression Predict Worse Prognosis for Cholangiocarcinoma Patients after Curative Resection
}

\author{
Attapol Titapun (D) ${ }^{1,2}$ \\ Vor Luvira ${ }^{1,2}$ \\ Tharatip Srisuk (iD) 1,2 \\ Apiwat Jareanrat ${ }^{1,2}$ \\ Vasin Thanasukarn (iD) 1,2 \\ Malinee Thanee ${ }^{2,3}$ \\ Prakasit Sa-Ngiamwibool ${ }^{2,3}$ \\ Sureerat Padthaisong ${ }^{2,4}$ \\ Kassaporn Duangkumpha ${ }^{2,4}$ \\ Manida Suksawat ${ }^{2,4}$ \\ Watcharin Loilome 2,4 \\ Paiboon Sithithaworn ${ }^{2,5}$ \\ Anchalee Techasen ${ }^{2,6}$ \\ Bandit Thinkhamrop ${ }^{2,7}$ \\ Alexa Dzienny ${ }^{8}$ \\ Ayse Caglayan ${ }^{9}$ \\ David Park (iD) ${ }^{9}$ \\ Simran Mahmud (iD) ${ }^{9}$ \\ Narong Khuntikeo (iD) 1,2
}

'Department of Surgery, Faculty of Medicine, Khon Kaen University, Khon Kaen, Thailand;

${ }^{2}$ Cholangiocarcinoma Research Institute (CARI), Khon Kaen University, Khon Kaen,

Thailand; ${ }^{3}$ Department of Pathology, Faculty of Medicine, Khon Kaen University, Khon Kaen, Thailand; ${ }^{4}$ Department of Biochemistry, Faculty of Medicine, Khon Kaen University, Khon Kaen, Thailand; ${ }^{5}$ Department of Parasitology, Faculty of Medicine, Khon Kaen University, Khon Kaen, Thailand; ${ }^{6}$ Faculty of Associated Medical

Sciences, Khon Kaen University, Khon Kaen, Thailand; ${ }^{7}$ Department of Epidemiology and Biostatistics, Faculty of Public Health, Khon Kaen University, Khon Kaen, Thailand; ${ }^{8}$ School of Medicine and Health Sciences, George

Washington University, Washington, DC, USA

${ }^{9}$ Faculty of Medicine, Imperial College London, London, UK

Correspondence: Attapol Titapun

Department of Surgery, Faculty of Medicine,

Khon Kaen University, Khon Kaen, Thailand

Tel +66-43-363252

Email attati@kku.ac.th
Background: Opisthorchis viverrini (OV)-associated cholangiocarcinoma (CCA) has a high immune response with chronic inflammation and oxidative stress. CD44 and Nestin, two cancer stem cell (CSC) markers, play major roles in cancer cell survival. Effects of immune response and expression CSC markers on survival of patients with CCA remain unclear.

Objective: To investigate the effects of level of OV IgG together with CSC marker expression and also the combination of these markers on survival of CCA patients after curative resection. Methods: All serum specimens from CCA patients who underwent curative surgery from 2005 to 2015 were examined for IgG for OV antigen by ELISA. Tissue specimens were studied for CD44 and Nestin expression. Survival analysis by Cox proportional hazard model was used for estimating hazard ratio (HR) with a 95\% confidence interval (CI).

Results: In this study, 122 (69.3\%) of 176 were positive for OV IgG, and 35 (19.9\%) were considered to have high-positive OV IgG. CD44s positive expression was found in $54(40 \%)$, CD44v6 high expression in 96 (69.6\%), CD44v8-10 high expression in $87(63.5 \%)$ and Nestin high expression in 21 (16.1\%). Multivariate survival analysis found that high-positive $\mathrm{OV} \mathrm{IgG} \mathrm{and} \mathrm{late} \mathrm{stage} \mathrm{tumor} \mathrm{were} \mathrm{independent} \mathrm{prognostic} \mathrm{factors} \mathrm{with} \mathrm{the} \mathrm{adjusted} \mathrm{HR} \mathrm{of}$ 2.24 (95\% CI 1.27-3.93) and 2.78 (95\% CI 1.46-5.29), respectively. Subgroup analysis in early and late stage CCA showed that a combined positive OV IgG and CD44s expression with the high expression of CD44v8-10 had the significantly poorest prognosis with HR of 3.75 (95\% CI 1.61-8.72) and HR of 1.76 (95\% CI 1.02-3.03), respectively.

Conclusion: A high level of OV IgG as well as a high level of CSC markers resulted in an aggressive CCA. OV IgG level together with CSC markers can be used as the prognostic markers for CCA patients' survival. The study of the CD44 pathway is promising for adjuvant treatment.

Keywords: CCA, OV-associated CCA, survival, OV IgG antibody, CD44s, CD44v8-10

\section{Introduction}

Cholangiocarcinoma (CCA) is a cancer of the bile duct epithelial cells arising along the intrahepatic or extrahepatic biliary tract. ${ }^{1}$ The north-eastern region of Thailand has the highest incidence of CCA in the world. The major risk factor for cholangiocarcinogenesis is chronic biliary tract inflammation caused by liver fluke, Opisthorchis viverrini (OV), infection. ${ }^{2}$ The mechanisms of inflammation and immunity that are involved in $\mathrm{OV}$ associated cholangiocarcinogenesis have been 
reported. ${ }^{3}$ Notably, chronic inflammation by OV infection can induce oxidative stress, which leads to abnormal tissue remodelling, DNA damage and an alteration in gene expression, all of which could promote CCA carcinogenesis. $^{3}$ OV associated CCA is mainly caused by the activation of inflammation, while the cause of non-OV associated CCA is related to the alteration of growth factors. ${ }^{4}$ Serum IgG against $\mathrm{OV}$ antigen is a good marker for OV associated CCA because it can represent past or present infection and is associated with a poor survival outcome. $^{5}$ The presence of serum IgG for OV is evidence of host immune response induced inflammation and the level of serum IgG may represent the level of oxidative stress that has occurred.

Cancer stem cells (CSC) are known to be a subpopulation of cancer cells with stem-like properties. They are involved in cancer development and progression. ${ }^{6}$ Cancer stem cell markers are therefore widely used as biomarkers in cancer patients. The cluster of differentiation 44 (CD44) antigen is a cell surface glycoprotein involved in cell-cell interactions, cell adhesion and migration that is important in many types of cancer and CD44 were also reported to be associated with shorter survival in various types of cancer. ${ }^{7-12}$ CD44 standard form (CD44s) and variant form (CD44v) overexpression was found in hyperplastic bile duct epithelium and cholangiocarcinoma cells in the OVinduced CCA hamster model. ${ }^{13}$ Several studies have investigated the role of CD44 in CCA. High expression of CD44 in the biliary epithelium indicates an unfavorable patient outcome. ${ }^{14}$ Another factor, Nestin, is a class VI intermediate filament protein which has been identified as a putative marker of CSC. ${ }^{15}$ Nestin expression has been observed in many types of human cancers. In some cancers, up-regulated or highly positive Nestin expression has been associated with a poor prognosis and tumor progression such as cell invasion, proliferation and metastasis. $^{16}$

CCA is reported to have a poor prognosis with 5-year survival rate after resection only $20-30 \%$ with high recurrence rate. ${ }^{17-20}$ Adjuvant treatment after surgery is not well established; many studies and several Phase III clinical trials have failed to address the benefit of adjuvant regimen of treatment. The recent BILCAP study reported that adjuvant treatment after surgery with capecitabine resulted in a significant increase in recurrent free survival compared with observation. $^{21}$ Although the results from the BILCAP study has changed the standard of care in adjuvant treatment for CCA and there are many ongoing trials focusing on adjuvant treatment for CCA, the use of specific risk factors or gene expression to select patients who may benefit from distinct treatments remain a challenge. $^{22}$ Even though there are studies demonstrating the prognostic effect of OV IgG level and CSC markers on CCA, the prognostic effect of serum OV IgG in the combination with cancer stem cell markers has not been elucidated. Therefore, in this study, we hypothesized that the combination of OV IgG level with cancer stem cell markers (CD44s, CD44v6, CD44v8-10, Nestin) is associated with poor survival, and that using these combined markers may enhance prognostic efficacy and selection of patients for specific adjuvant treatment.

\section{Materials and Methods}

\section{Patients and Serums}

CCA patients who were diagnosed at Srinagarind Hospital, Khon Kaen University (January 1, 2005 to December 31, 2017) were included in this study. Informed consent had been obtained from all patients before surgery for usage of excessive tissue and serum samples. Serum specimens were kept in the biobank of the Cholangiocarcinoma Research Institute (CARI), Khon Kaen University. Patients who had undergone palliative surgery or had inadequate serum specimens were excluded. The clinicopathological information was provided by the CARI. The patient's age, gender, tumor site, histology type, staging, lymph node metastatic status, surgical margin status and adjuvant chemotherapy were recorded. Patient survival was defined as the interval from the day of surgery to the time of death. A patient who was alive at the end of the study on December 31 , 2019 were recorded as censor. This research protocol was approved by the Human Research Ethics Committee, Khon Kaen University, based on the Declaration of Helsinki and the ICH Good Clinical Practice Guidelines (HE621147).

\section{Indirect Enzyme Linked Immunosorbent Assay (ELISA)}

Immunoglobulin $\mathrm{G}$ ( $\mathrm{IgG}$ ) antibodies against OV antigen were investigated in CCA serum using the indirect ELISA method. Briefly, whole adult OV antigen $(1500 \mu \mathrm{g} / \mathrm{mL}$ in $1 \mathrm{X}$ PBS $\mathrm{pH}$ 7.4) was coated into flat bottom microtiter plates that were then incubated at $4{ }^{\circ} \mathrm{C}$ overnight. Before blocking with $3 \%$ skimmed milk in PBS $(250 \mu \mathrm{L} /$ well $)$ at 
$37^{\circ} \mathrm{C}$ for $1 \mathrm{~h}$, the plates were washed with $1 \mathrm{X}$ PBS with $0.05 \%$ Tween 20. After that, patient serum (dilution $1: 6000$ in $3 \%$ skimmed milk) was added into the plate at $100 \mu \mathrm{L} /$ well in duplicate, then incubated at $4^{\circ} \mathrm{C}$ overnight. After washing with PBS plus Tween 20 five times, $100 \mu \mathrm{L}$ of goat anti-human IgG conjugated with horseradish peroxidase (dilution 1:3000 in 3\% skimmed milk) was added and incubated at $37^{\circ} \mathrm{C}$ for $2 \mathrm{~h}$. After washing with PBS plus Tween 20, the signal was developed by adding orthophenylene diamine hydrochloride (OPD) (Zymed, CA, USA) substrate (100 $\mu \mathrm{L} /$ well) and incubated for $30 \mathrm{~min}$. $4 \mathrm{~N}$ sulfuric acid was used to stop the reaction and the optical density (OD) was measured using an ELISA reader (at $492 \mathrm{~nm}$ ) (Tecan, Austria). The results were analysed as described in a previous study. ${ }^{23}$ Patients with positive OV $\operatorname{IgG}$ and arbitrary units (AU) above percentile 75 were considered high titer positive.

\section{CCA Tissue Microarray (TMA)}

Of the total of $176 \mathrm{CCA}$ cases, there were 163 available sera matched CCA tissue sections observed at selected tumor regions and normal liver tissue for negative controls. A manual tissue microarrayer $(2.0 \mathrm{~mm}$ diameter needle) was used to prepare the TMA block. This was produced with 70 cores per block. TMA blocks were cut into $4 \mu \mathrm{m}$ thick sections and mounted on coated glass slides.

\section{Antibodies}

Primary antibodies in this study were as follows: mouse anti-Nestin (\#ab6320), mouse anti-CD44 (\#ab16728), mouse anti-CD44v6 (\#ab78960), anti-rat (\#ab6734) (Abcam, Cambridge, MA, USA). Rat anti-CD44v8-10 (\#LKG-M001, Cosmo Bio, Tokyo, Japan).

\section{Immunohistochemistry (IHC)}

CCA TMAs were used to investigate the expression of the target proteins using IHC. Briefly, paraffin embedded CCA TMAs were deparaffinized and rehydrated in stepwise xylene followed by $100 \%, 90 \%, 80 \%$ and $70 \%$ ethanol. Antigen retrieval was performed using microwave cooking with $1 \mathrm{mM}$ sodium citrate $\mathrm{pH} 6.0$ plus $0.05 \%$ Tween 20 for $10 \mathrm{~min}$. The activity of endogenous peroxide and nonspecific binding were blocked using $0.3 \%$ (v/v) of hydrogen peroxide and $10 \%$ skim milk for 10 minutes for each. Primary antibodies including anti-Nestin (dilution 1:50), anti-CD44 (dilution 1:25), anti-CD44v6 (dilution 1:50) and anti-CD44v8-10 (dilution 1:50) were added and incubated at room temperature for $1 \mathrm{~h}$ followed by $4^{\circ} \mathrm{C}$ overnight. The sections were washed with phosphate buffer saline (PBS) with $0.1 \%$ Tween 20 for 5 min (three times) followed by PBS for $5 \mathrm{~min}$. The Envision horseradish peroxidase conjugated secondary antibody (Dako, USA) was added in TMA for $1 \mathrm{~h}$, except for the CD44v810 tissue section to which was added anti-rat antibody (dilution 1:100) and left for $3 \mathrm{~h}$. After washing, the signal was developed using a 3,3' diaminobenzidine tetrahydrochloride (DAB) substrate kit (Vector Laboratories, Inc., CA) for $10 \mathrm{~min}$ and counterstained with Mayer's hematoxylin for $5 \mathrm{~min}$. Before mounting, the sections were dehydrated with stepwise $70 \%, 80 \%, 90 \%, 100 \%$ ethanol and xylene, for 5 min each. The TMAs were observed under a light microscope.

\section{IHC Scoring System}

Protein expression was analysed according to the frequency and intensity scores. The staining frequency score of the target protein was semi-quantitatively scored and classified as 0 if there was no positive staining, $1+=$ less than $25 \%$ of positive cells, $2+=25-50 \%$ of positive cells and $3+=$ more than $50 \%$ of positive cells. The intensity score was defined as 0 if there was no staining, $1+=$ weak staining, $2+=$ moderate staining and $3+=$ strong staining. The final IHC score was calculated by multiplying the intensity score with the frequency score. The IHC scores ranged between $0-9$. The IHC score was formed from two independent punctures of each patient which were used to calculate the average IHC score. Finally, the median value, which is calculated from all patients, was used as the cutoff value to classify low and high expression.

\section{Statistical Analysis}

The sample size was determined for single or binary covariate cox regression analysis methods proposed by Schmoor et al. ${ }^{24}$ Setting a power of $80 \%$ at a 0.05 significance level, aiming to detect a hazard ratio of 2.0, assuming a prevalence of IgG for OV positive of 0.75 and prevalence of $\mathrm{CD} 44$ positive $\mathrm{CCA}$ of 0.8 , and a correlation coefficient between IgG for OV and CD44 positive of 0 , the sample size was 110 cases which allowed for the expected data loss of $10 \%$. Continuous and categorical data were reported as mean \pm standard deviation (SD) and percentages, respectively. The Kaplan-Meier survival analysis was used to obtain median survival time with a 95\% confidence interval (CI). Cox proportional hazard model was used to estimate the hazard ratio 
(HR) with a 95\% CI as well as p-values. Stata version 14 and SPSS version 17 were used to analyse the data. Significance level was set at 0.05 .

\section{Results}

\section{Patients' Characteristics and} Histopathology

A total of 221 histologically confirmed CCA patients who underwent surgery are included in this study. The biopsy data of 45 patients who underwent palliative surgery were excluded. Among the 176 patients included, the mean age of patients was 59.6 years (age ranged between 34 and 82 years), and $108(61.4 \%)$ patients were male. Fifty-three (30\%) patients were extrahepatic, 97 (55.1\%) had papillary carcinoma, 55 (31.2\%) had tubular carcinoma and 24 (13.7\%) patients had non-specific adenocarcinoma and rare variants. Tumor staging was defined according to the American Joint Committee on Cancer Staging manual, 8th edition. Stage 0, I, II, III and IV were found in $11(6.3 \%), 27(15.3 \%), 27$ (15.3\%), $103(58.5 \%)$ and $8(4.6 \%)$ patients, respectively. Lymph node metastasis was found in 92 (52.3\%) cases. A negative histological margin was achieved in 96 (54.5\%) cases; $80(45.5 \%)$ cases showed a microscopically positive margin (R1). Fifty-three (30\%) patients received adjuvant chemotherapy after surgical resection (Table 1).

The 176 CCA patients who received curative surgery were studied for serum OV IgG antibody. Of these, 54 (30.7\%) cases were negative for OV $\mathrm{IgG}$, while 122 (69.3\%) cases were positive. Among these 122 patients, 35

Table I Patient Characteristics by Level of OV IgG

\begin{tabular}{|c|c|c|c|c|c|}
\hline \multirow[t]{2}{*}{ Variables } & \multirow[t]{2}{*}{ Overall } & \multicolumn{3}{|c|}{ Level of OV IgG } & \multirow[t]{2}{*}{ p-value } \\
\hline & & Negative & Low-Positive & High-Positive & \\
\hline Overall & 176 & 54 (30.7\%) & 87 (49.4\%) & 35 (19.9\%) & \\
\hline Age (mean, year) & 59.6 & 59.1 & 60.1 & 59.4 & \\
\hline $\begin{array}{l}\text { Gender } \\
\text { Female } \\
\text { Male }\end{array}$ & $\begin{array}{l}68(38.6 \%) \\
108(61.4 \%)\end{array}$ & $\begin{array}{l}23(42.6 \%) \\
31 \text { (57.4\%) }\end{array}$ & $\begin{array}{l}37(42.5 \%) \\
50(57.5 \%)\end{array}$ & $\begin{array}{c}8(22.8 \%) \\
27(77.2 \%)\end{array}$ & 0.10 \\
\hline $\begin{array}{l}\text { Tumor site } \\
\text { Intrahepatic } \\
\text { Extrahepatic }\end{array}$ & $\begin{array}{l}123(70 \%) \\
53(30 \%)\end{array}$ & $\begin{array}{c}36(66.7 \%) \\
18(33.3)\end{array}$ & $\begin{array}{l}64(73.6 \%) \\
23(26.4 \%)\end{array}$ & $\begin{array}{l}23(65.7 \%) \\
12(34.3 \%)\end{array}$ & 0.57 \\
\hline $\begin{array}{l}\text { Histology } \\
\text { Papillary } \\
\text { Non-papillary }\end{array}$ & $\begin{array}{l}97(55.1 \%) \\
79(44.9 \%)\end{array}$ & $\begin{array}{l}33(61.1 \%) \\
21 \text { (38.9\%) }\end{array}$ & $\begin{array}{l}45(51.7 \%) \\
42(48.3 \%)\end{array}$ & $\begin{array}{l}19(54.3 \%) \\
10(45.7 \%)\end{array}$ & 0.35 \\
\hline $\begin{array}{c}\text { Stage } \\
0 \\
\text { I } \\
\text { II } \\
\text { III } \\
\text { IV }\end{array}$ & $\begin{array}{l}\text { II (6.3\%) } \\
27(15.3 \%) \\
27(15.3 \%) \\
103(58.5 \%) \\
8(4.6 \%)\end{array}$ & $\begin{array}{c}7(13 \%) \\
7(13 \%) \\
9(16.6 \%) \\
29(53.7 \%) \\
2(3.7 \%)\end{array}$ & $\begin{array}{c}4(4.6 \%) \\
15(17.2 \%) \\
14(16.1 \%) \\
50(57.5 \%) \\
4(4.6 \%)\end{array}$ & $\begin{array}{c}0 \\
5(14.3 \%) \\
4(11.4 \%) \\
24(68.5 \%) \\
2(5.7 \%)\end{array}$ & 0.39 \\
\hline $\begin{array}{l}\text { Lymph node metastasis } \\
\text { No } \\
\text { Yes }\end{array}$ & $\begin{array}{l}84(47.7 \%) \\
92(52.3 \%)\end{array}$ & $\begin{array}{l}31 \text { ( } 57.4 \%) \\
23(42.6 \%)\end{array}$ & $\begin{array}{l}4 I \text { ( }(47.1 \%) \\
46(52.9 \%)\end{array}$ & $\begin{array}{l}12(34.3 \%) \\
23(65.7 \%)\end{array}$ & 0.10 \\
\hline $\begin{array}{l}\text { Surgical margin } \\
\text { Negative } \\
\text { Positive }\end{array}$ & $\begin{array}{l}96(54.5 \%) \\
80(45.5 \%)\end{array}$ & $\begin{array}{l}12(48 \%) \\
13(52 \%)\end{array}$ & $\begin{array}{l}22(40 \%) \\
33(60 \%)\end{array}$ & $\begin{array}{l}9(56.3 \%) \\
7(43.7 \%)\end{array}$ & 0.48 \\
\hline $\begin{array}{l}\text { Adjuvant chemotherapy } \\
\text { Yes } \\
\text { No }\end{array}$ & $\begin{array}{l}53(30 \%) \\
123(70 \%)\end{array}$ & $\begin{array}{l}13(24 \%) \\
41(76 \%)\end{array}$ & $\begin{array}{l}33(37.9 \%) \\
54(62.1 \%)\end{array}$ & $\begin{array}{c}7(20 \%) \\
28(80 \%)\end{array}$ & 0.07 \\
\hline
\end{tabular}




\section{CD44s}
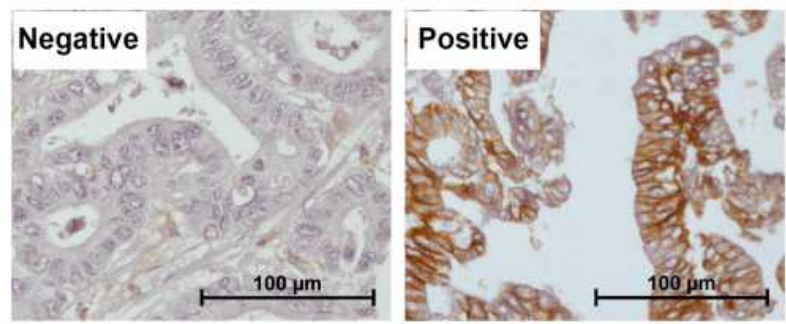

CD44v8-10
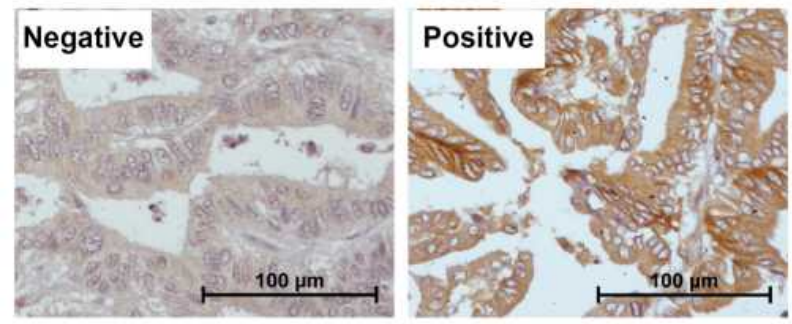

\section{CD44v6}

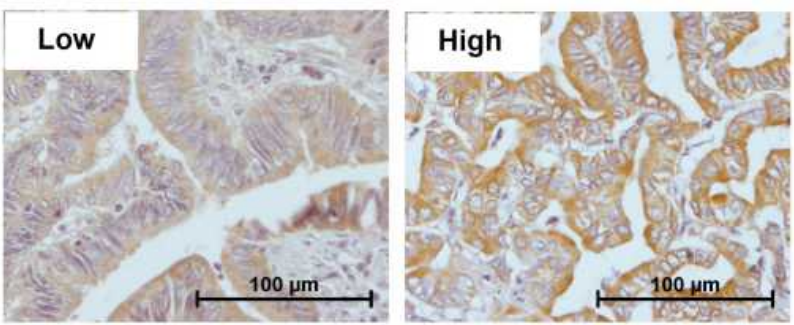

Nestin

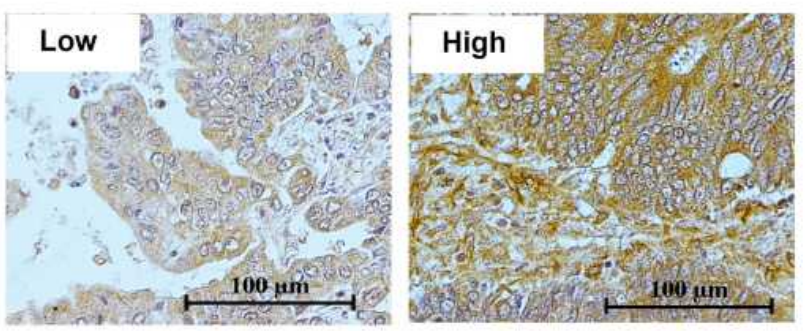

Figure I Representative IHC staining of CD44s, CD44v6, CD44v8-10 and Nestin in human CCA tissues (magnification 20X).

(19.9\%) were considered to have a high level of OV IgG. In addition, the expression of CD44s, CD44v6, CD44v8-10 and Nestin were investigated in 176 CCA tissues. IHC staining in CCA tissue was demonstrated (Figure 1). For CD44s, 54 (40\%) cases were classified as positive. For CD44 variant, CD44v6 showed high expression in $96(69.6 \%)$ cases and
CD44v8-10 in 87 (63.5\%) cases. A high expression of Nestin was found in $21(16.1 \%)$ cases. The association between histopathology factors and IHC results was explored according to OV IgG levels. There was no difference in the histopathology factors and IHC results between OV IgG negative, positive and high-positive groups (Table 2).

Table 2 The Expression of CD44s, CD44v6, CD44v8-10 and Nestin by Level of OV IgG

\begin{tabular}{|c|c|c|c|c|c|}
\hline \multirow[t]{2}{*}{ Variables } & \multirow[t]{2}{*}{ Overall } & \multicolumn{3}{|c|}{ Level of OV IgG } & \multirow[t]{2}{*}{ p-value } \\
\hline & & Negative & Low-Positive & High-Positive & \\
\hline Overall & 176 & 54 (30.7\%) & 87 (49.4\%) & 35 (19.9\%) & \\
\hline CD44 & 135 & $4 I$ & 67 & 27 & 0.98 \\
\hline Negative & 81 (60\%) & $25(61 \%)$ & 40 (59.7\%) & 16 (59.3\%) & \\
\hline Positive & 54 (40\%) & $16(39 \%)$ & 27 (40.3\%) & II (40.7\%) & \\
\hline CD44v6 & 138 & 43 & 68 & 27 & 0.59 \\
\hline Low & $42(30.4 \%)$ & II (25.6\%) & 21 (30.9\%) & $10(37 \%)$ & \\
\hline High & $96(69.6 \%)$ & $32(74.4 \%)$ & 47 (69.1\%) & 17 (63\%) & \\
\hline CD44v8 & 137 & 43 & 67 & 27 & 0.37 \\
\hline Low & 50 (36.5\%) & 12 (27.9\%) & 27 (40.3\%) & II (40.7\%) & \\
\hline High & 87 (63.5\%) & 31 (72.1\%) & 40 (59.7\%) & 16 (59.3\%) & \\
\hline Nestin & 130 & 40 & 64 & 26 & 0.65 \\
\hline Low & $109(83.9 \%)$ & $32(80 \%)$ & 54 (84.4\%) & $23(88.5 \%)$ & \\
\hline High & $21(16.1 \%)$ & $8(20 \%)$ & $10(15.6 \%)$ & 3 (1I.5\%) & \\
\hline
\end{tabular}


A

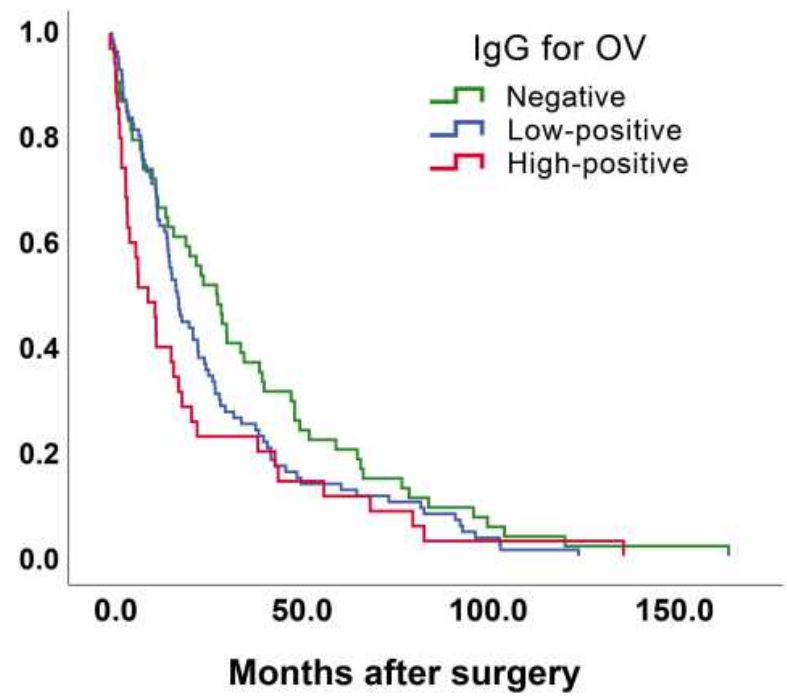

B

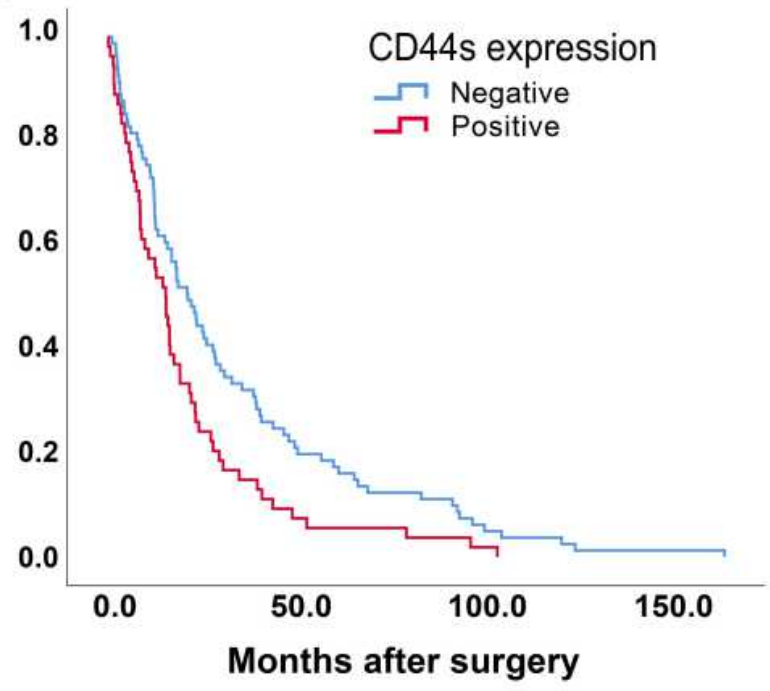

Number at risk

Negative 81

Positive 54

$\begin{array}{lll}18 & 5 & 1 \\ 5 & 1 & 0\end{array}$

\begin{tabular}{ccrcc}
\multicolumn{2}{c}{ Number at risk } & & & \\
Negative & 54 & 16 & 4 & 1 \\
Positive & & & & \\
Low & 87 & 14 & 3 & 0 \\
High & 35 & 5 & 1 & 0
\end{tabular}

Figure 2 Kaplan-Meier analysis of serum IgG for OV and CD44s expression in 176 CCA patients. (A) Survival by different levels of serum IgG for OV. (B) Survival by different levels of CD44s.

\section{Survival Analysis by Level of Serum OV IgG, Histopathological Factors and IHC Results (CD44s, CD44v6, CD44v8-I0, Nestin)}

The overall median survival time (MST) after curative resection was 18.6 months and 1-year, 3-year and 5-year survival rates were $67.0 \%$ (95\% CI 59.6-73.4), 30.3\% (95\% CI 23.6-37.2) and 21.2\% (95\% CI 15.3-27.7), respectively.

Bivariable analysis showed that CCA patients with a lowpositive OV IgG had a poor prognosis with an MST of 18.6 months (95\% CI 9.2-16.0) and an HR of 1.45 (95\% CI 0.98-2.15, $\mathrm{p}=0.06$ ). Patients with high-positive OV IgG had a significantly shorter survival with an MST of 10.5 months (95\% CI 4.8-17.4) and an HR of 1.99 (95\% CI 1.24-3.19) compared with OV IgG negative patients (Figure 2A). Other factors that had a significant influence on survival were positive resection margin (HR 2.28, 95\% CI 1.63-3.19, $\mathrm{p}<0.01$ ), papillary type (HR $0.56,95 \%$ CI $0.40-0.68, \mathrm{p}<$ 0.01 ), lymph node metastasis (HR 3.73,95\% CI 2.58-5.39, $\mathrm{p}<0.01$ ) and late stage tumor (HR 4.21, 95\% CI 2.85-6.23, $\mathrm{p}<0.01$ ). For IHC results, the positive expression of CD44s had a significant influence on survival (HR 1.52, 95\% CI 1.04-2.23, p = 0.03) (Figure 2B) (Table 3). Multivariate analysis showed that only high-positive OV $\operatorname{IgG}$ and late stage tumor were independent prognostic factors with an adjusted HR of 2.24 (95\% CI 1.27-3.93, p < 0.01) and 2.78 (95\% CI 1.46-5.29, p < 0.01), respectively (Table 3).

\section{Survival Analysis by Level of Serum OV IgG, Histopathological Factors and IHC Results (CD44s, CD44v6, CD44v8-I0, Nestin) According to Tumor Stage}

It is possible that the strong effect of tumor stage on patient survival may obscure the effect of other factors on survival outcome, therefore, survival based on $\mathrm{OV}$ IgG level, CD44, CD44v6, CD44v8-10 and Nestin expression was analysed according to tumor stage grouping at an early and late stage.

In early stage CCA (stages $0, \mathrm{I}, \mathrm{II}$ ), a positive expression of CD44s showed a poorer prognosis, with an HR of 2.31 (95\% CI 1.25-4.26, $\mathrm{p}=0.008)$ compared with negative expression (Table 4) (Supplementary Figure 2A). On the other hand, there was no difference in prognosis found based on OV IgG level (Supplementary Figure 1), CD44v6, CD44v8-10 and Nestin expression (Supplementary Figure 2B-D). Survival analysis based 
on the combination of $\mathrm{OV} \operatorname{IgG}$ level and protein expression was also explored. The results with significant outcomes for survival were CD44s positive expression with OV IgG positive (HR 2.45, 95\% CI 1.22-4.95, $\mathrm{p}=0.012$ ),
CD44v8-10 high expression with OV IgG positive (HR 2.10, 95\% CI 1.14-3.87, $\mathrm{p}=0.018$ ), CD44s positive expression with CD44v8-10 high expression (HR 3.27, 95\% CI 1.68-6.36, p < 0.001) and OV IgG positive

Table 3 Median Survival Time and Multivariate Analyses of Clinicopathological Data and CSC Markers

\begin{tabular}{|c|c|c|c|c|c|c|}
\hline Variables & $\mathbf{N}$ & IR/I00 & MST, Months (95\% Cl) & Crude HR $(95 \% \mathrm{Cl})$ & Adjusted HR $(95 \% \mathrm{Cl})$ & p-value \\
\hline Overall & 176 & 2.7 & I8.6 (I5.8-28.9) & & & \\
\hline \multicolumn{7}{|l|}{ Level of OV IgG } \\
\hline Negative & 54 & 1.9 & $29.2(15.9-42.1)$ & I & I & \\
\hline Low-positive & 87 & 2.9 & I8.6 (I5.6-24.2) & $1.45(0.98-2.15)$ & $1.50(0.95-2.37)$ & 0.08 \\
\hline High-positive & 35 & 4.0 & $10.5(4.8-17.4)$ & $1.99(1.24-3.19)$ & $2.24(1.27-3.93)$ & $<0.01 *$ \\
\hline \multicolumn{7}{|l|}{ Gender } \\
\hline Female & 68 & 2.8 & $16.3(12.8-28.8)$ & 1 & - & - \\
\hline Male & 108 & 2.6 & I8.9 (I5.6-24.2) & $0.97(0.69-1.36)$ & & \\
\hline \multicolumn{7}{|l|}{ Tumor site } \\
\hline Intrahepatic & 123 & 2.7 & I7.4 (I3.I-23.6) & 1 & - & - \\
\hline Extrahepatic & 53 & 2.7 & $20.8(15.3-29.5)$ & $0.97(0.68-1.39)$ & & \\
\hline \multicolumn{7}{|l|}{ Histology } \\
\hline Non-papillary & 79 & 4.1 & $13.0(8.9-16.3)$ & I & I & \\
\hline Papillary & 97 & 2.0 & $26.9(19.7-33.8)$ & $0.56(0.40-0.68)$ & $0.72(0.49-1.06)$ & 0.10 \\
\hline \multicolumn{7}{|l|}{ Stage } \\
\hline $0, \mathrm{I}, \mathrm{II}$ & 65 & 1.1 & $51.8(35.7-123.9)$ & 1 & 1 & \\
\hline III, IV & 111 & 5.5 & $12.7(9.2-15.9)$ & $4.21(2.85-6.23)$ & $2.78(1.46-5.29)$ & $<0.01 *$ \\
\hline \multicolumn{7}{|l|}{ Lymph node metastasis } \\
\hline No & 84 & 1.4 & $41.5(28.1-63.0)$ & 1 & 1 & \\
\hline Yes & 92 & 6.2 & $12.5(8.5-\mid 5.9)$ & $3.73(2.58-5.39)$ & I.53 $(0.86-2.7 I)$ & 0.14 \\
\hline \multicolumn{7}{|l|}{ Resection margin } \\
\hline Negative & 96 & 1.9 & $30.2(2|.8-4| .5)$ & I & I & \\
\hline Positive & 80 & 4.5 & $12.7(8.9-16.3)$ & $2.28(1.63-3.19)$ & I.I5 (0.76-I.72) & 0.50 \\
\hline \multicolumn{7}{|l|}{ Adjuvant chemotherapy } \\
\hline Yes & 53 & 2.8 & $19.2(\mid 3.7-25.9)$ & I & - & - \\
\hline No & 123 & 2.6 & I8.I (I0.1-24.2) & $1.06(0.75-1.5)$ & & \\
\hline \multicolumn{7}{|l|}{ CD44 } \\
\hline Negative & 81 & 2.4 & $22.8(15.6-29.5)$ & 1 & I & \\
\hline Positive & 54 & 4.2 & $15.8(8.9-18)$ & $1.52(1.04-2.23)$ & $\mathrm{I} .43(0.97-2.1 \mathrm{I})$ & 0.07 \\
\hline \multicolumn{7}{|l|}{ CD44v6 } \\
\hline Low & 42 & 3.1 & I9.7 (II.I-24) & 1 & - & - \\
\hline High & 96 & 2.7 & $17.0(13.2-24.9)$ & $0.95(0.64-1.42)$ & & \\
\hline \multicolumn{7}{|l|}{ CD44v8-10 } \\
\hline Low & 50 & 3.0 & $17.4(\mid 2.5-24.9)$ & I & - & - \\
\hline High & 87 & 2.7 & $18.6(13.2-24)$ & $0.90(0.62-1.32)$ & & \\
\hline \multicolumn{7}{|l|}{ Nestin } \\
\hline Low & 109 & 2.9 & $16.8(|2.9-2| .8)$ & 1 & - & - \\
\hline High & 21 & 2.2 & $26.2(9.2-35.9)$ & $0.78(0.46-1.33)$ & & \\
\hline
\end{tabular}

Note: *P value equal to or less than 0.05 was considered statistically significant.

Abbreviations: $\mathrm{N}$, number of patients; HR, hazard ratio; IR/I00, incidence rate per 100 persons-month; MST, median survival time. 
together with CD44s positive expression and CD44v8-10 high expression (HR 4.39, 95\% CI 1.90-10.14, p = 0.001) (Table 4) (Figure 3). Interestingly, multivariate analysis showed that a combination of OV IgG level and protein expression were independent prognostic factors for patient survival. The worst prognostic value was found with the combination of OV IgG positive and positive CD44s and high CD44v8-10 expression with an adjusted HR of 3.75 (95\% CI 1.61-8.72, $\mathrm{p}=0.002$ ) (Table 5).
In late stage (stages III, IV) CCA, bivariate analysis showed that a positive resection margin and no adjuvant chemotherapy led to a poor prognosis with a crude HR of 1.59 (95\% CI 1.08-2.35) and 1.50 (95\% CI 1.00-2.24), respectively. For IHC factors, only the combination of $\mathrm{OV}$ IgG positive together with CD44s positive expression and CD44v8-10 high expression showed a significantly poor prognosis with a crude HR of 1.74 (95\% CI 1.01-2.98, $\mathrm{p}=$ $0.044)$, while there was no effect on survival outcome by

Table 4 Survival Analysis by Level of Serum OV IgG, Clinicopathological Factors and IHC Results (CD44s, CD44v6, CD44v8-I0, Nestin) According to Tumor Stage

\begin{tabular}{|c|c|c|c|c|c|c|}
\hline \multirow[t]{2}{*}{ Variables } & \multirow[b]{2}{*}{$\mathbf{N}$} & \multicolumn{2}{|l|}{ Early Stage } & \multirow[b]{2}{*}{$\mathbf{N}$} & \multicolumn{2}{|l|}{ Late Stage } \\
\hline & & Crude HR $(95 \% \mathrm{Cl})$ & p-value & & Crude HR $(95 \% \mathrm{Cl})$ & p-value \\
\hline $\begin{array}{l}\text { Gender (Ref: Female) } \\
\text { Male }\end{array}$ & 65 & $0.82(0.49-1.38)$ & 0.447 & 111 & $1.03(0.70-1.50)$ & 0.901 \\
\hline $\begin{array}{l}\text { Tumor site (Ref: Intrahepatic) } \\
\text { Extrahepatic }\end{array}$ & 65 & $1.05(0.59-1.56)$ & 0.866 & 111 & $0.78(0.52-1.16)$ & 0.211 \\
\hline $\begin{array}{l}\text { Histology (Ref: Non-papillary) } \\
\text { Papillary }\end{array}$ & 65 & $0.55(0.32-0.92)$ & $0.024 *$ & 111 & $0.70(0.48-1.03)$ & 0.069 \\
\hline $\begin{array}{l}\text { Resection margin (Ref: Negative) } \\
\text { Positive }\end{array}$ & 65 & $0.97(0.54-1.75)$ & 0.929 & III & $1.59(1.08-2.35)$ & $0.018^{*}$ \\
\hline $\begin{array}{l}\text { Adjuvant chemotherapy (Ref: Yes) } \\
\text { No }\end{array}$ & 65 & $1.25(0.69-2.26)$ & 0.455 & III & $1.50(1.00-2.24)$ & $0.048^{*}$ \\
\hline $\begin{array}{l}\text { Level of OV lgG (Ref: Negative) } \\
\text { Low-positive } \\
\text { High-positive }\end{array}$ & 65 & $\begin{array}{l}1.4 \mathrm{I}(0.82-2.44) \\
2.01(0.91-4.46)\end{array}$ & $\begin{array}{l}0.212 \\
0.085\end{array}$ & 111 & $\begin{array}{l}1.12(0.72-1.75) \\
1.36(0.79-2.32)\end{array}$ & $\begin{array}{l}0.607 \\
0.269\end{array}$ \\
\hline $\begin{array}{l}\text { CD44s (Ref: Negative) } \\
\text { Positive }\end{array}$ & 47 & $2.31(1.25-4.26)$ & $0.008^{*}$ & 88 & $1.20(0.78-1.84)$ & 0.416 \\
\hline $\begin{array}{l}\text { CD44v6 (Ref: Low) } \\
\text { High }\end{array}$ & 49 & $0.83(0.45-1.54)$ & 0.557 & 89 & $0.86(0.55-1.36)$ & 0.524 \\
\hline CD44v8-10 (Ref: Low) & 49 & & & 88 & & \\
\hline $\begin{array}{l}\text { High } \\
\text { Nestin (Ref: Low) } \\
\text { High }\end{array}$ & 46 & $\begin{array}{l}\text { I.4I (0.74-2.68) } \\
0.79(0.76-2.29)\end{array}$ & $\begin{array}{l}0.292 \\
0.669\end{array}$ & 83 & $\begin{array}{l}1.00(0.65-1.54) \\
0.58(0.32-1.04)\end{array}$ & $\begin{array}{l}0.989 \\
0.066\end{array}$ \\
\hline $\begin{array}{l}\text { CD44s and OV IgG } \\
\text { Positive }\end{array}$ & 47 & $2.45(1.22-4.95)$ & $0.012^{*}$ & 88 & $1.53(0.96-2.46)$ & 0.077 \\
\hline $\begin{array}{l}\text { CD44v8-10 and OV } \operatorname{lgG} \\
\text { High and Positive }\end{array}$ & 49 & $2.10(1.14-3.87)$ & $0.018^{*}$ & 88 & $1.41(0.91-2.19)$ & 0.120 \\
\hline $\begin{array}{l}\text { CD44s and CD44v8-10 } \\
\text { Positive and High }\end{array}$ & 47 & $3.27(1.68-6.36)$ & $<0.00 I^{*}$ & 88 & I. $27(0.78-2.05)$ & 0.336 \\
\hline $\begin{array}{l}\text { OV IgG and CD44s and CD44v8-10 } \\
\text { Positive and Positive and High }\end{array}$ & 47 & $4.39(1.90-10.14)$ & $0.00 I^{*}$ & 88 & $1.74(1.01-2.98)$ & $0.044^{*}$ \\
\hline
\end{tabular}

Notes: *P value equal to or less than 0.05 was considered statistically significant. Early stage, tumor stage 0-II; late stage, tumor stage III-IV.

Abbreviations: HR, hazard ratio. 


\section{A}
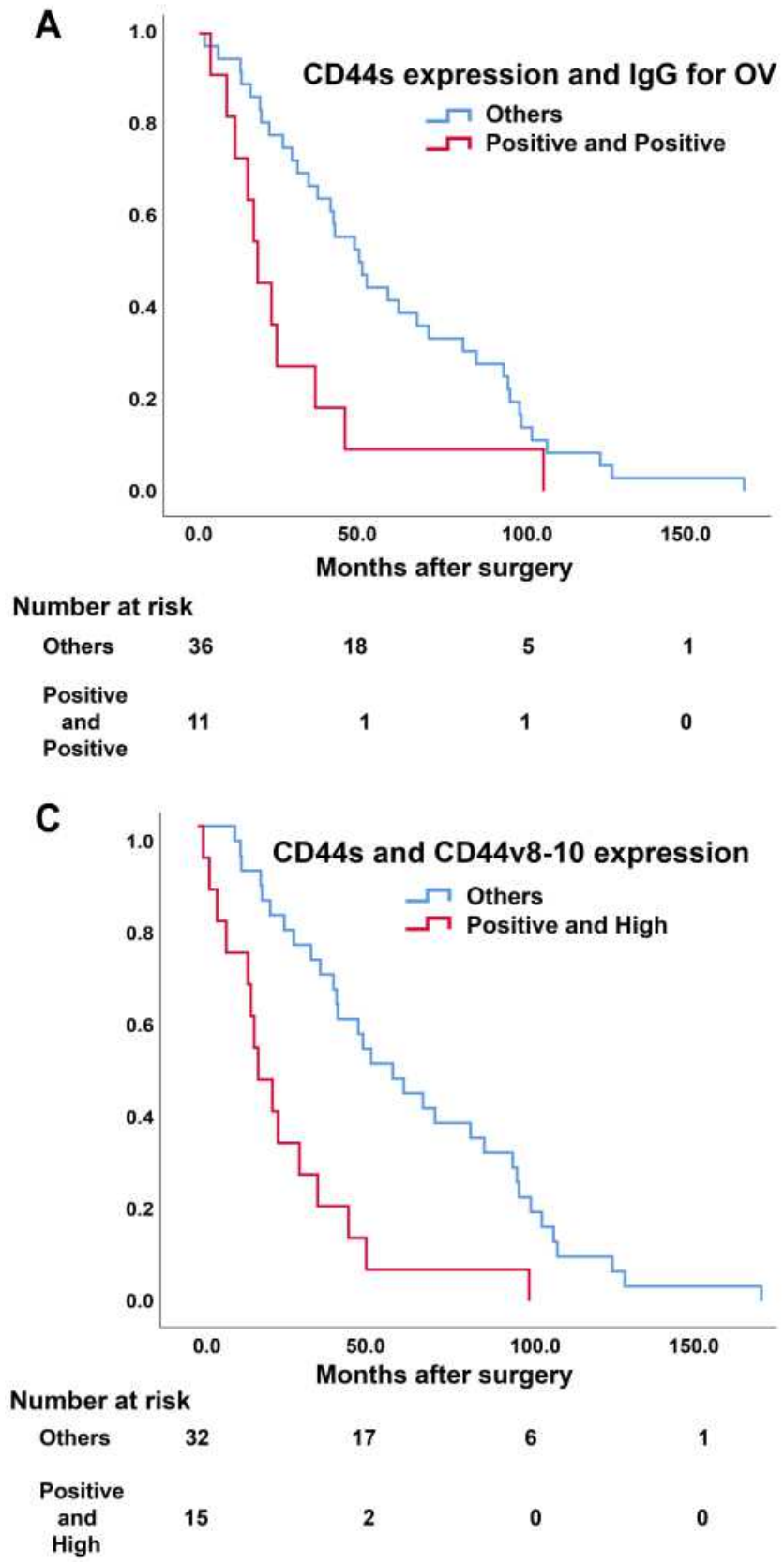

B

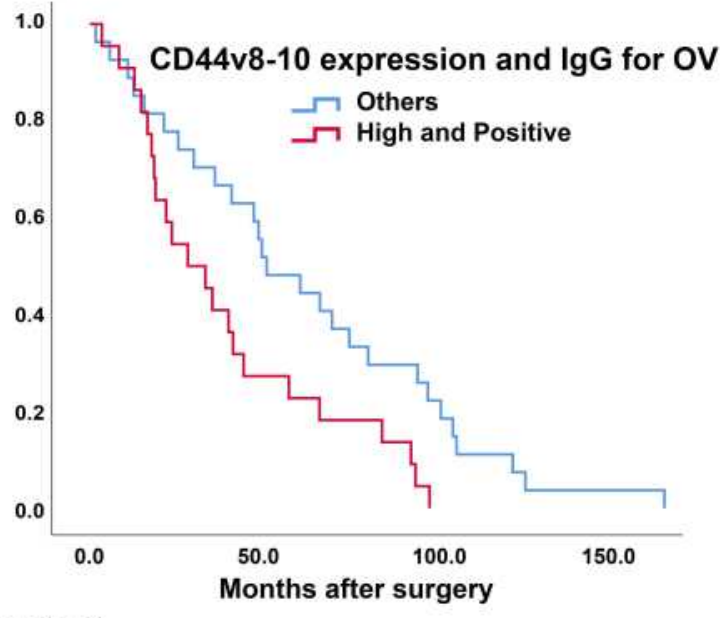

Number at risk

Others 27

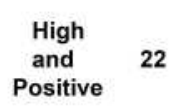

15

6

6

0

1

D

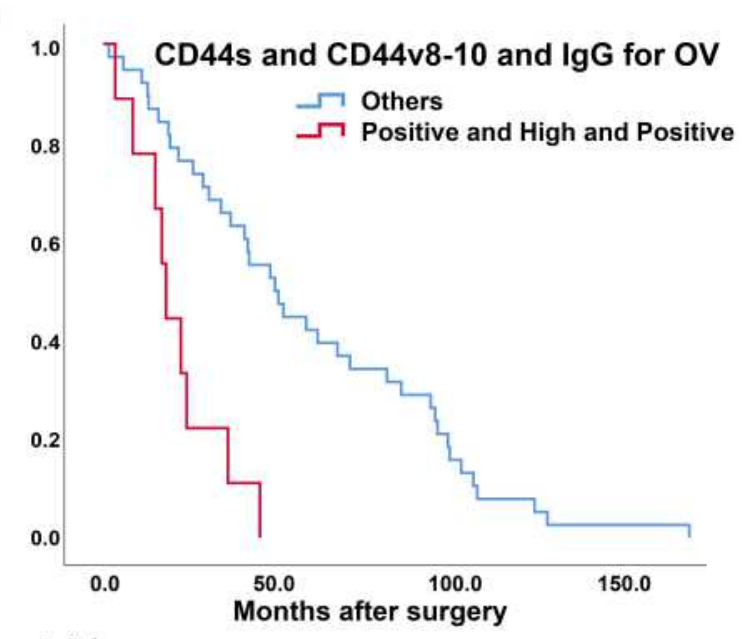

Number at risk

Others 38
Positive/High/
Positive 9

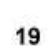

19

0

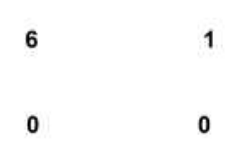

Figure 3 Kaplan-Meier analysis of overall survival with a combination of serum lgG for OV and IHC results in early stage CCA. (A) Survival with a combination of CD44s and $\operatorname{lgG}$ for OV. (B) Survival with a combination of CD44v8-10 and IgG for OV. (C) Survival with a combination of CD44s and CD44v8-10. (D) Survival with a combination of CD44s, CD44v8-10 and lgG for OV.

using one factor alone or the combination of two factors (Table 4) (Supplementary Figure 3 and 4, Figure 4). Multivariable analysis showed no adjuvant chemotherapy and the combination of OV IgG positive together with CD44s positive expression and CD44v8-10 high expression led to a significantly poorer survival with an adjusted HR of $1.72(95 \%$ CI 1.08-2.76, p= 0.023) and $1.76(95 \%$ CI $1.02-3.03, \mathrm{p}=0.041$ ), respectively (Table 6).

\section{Discussion}

Thailand has the highest incidence of CCA in the world ${ }^{25}$ and the major risk factor is $\mathrm{OV}$ infection. A previous study in 1994 reported that up to $89 \%$ of CCA patients were positive for serum OV IgG. $^{26}$ The current study had a lower prevalence of CCA patients with positive serum OV IgG at $70 \%$. As the current study included only patients who underwent curative resection, this may not 
Table 5 Multivariable Analysis of IHC Results in Early Stage CCA

\begin{tabular}{|c|c|c|c|}
\hline \multicolumn{4}{|l|}{ Early Stage (0, I, II) } \\
\hline Variables & $\mathbf{N}$ & $\begin{array}{c}\text { Adjusted HR } \\
(95 \% \mathrm{Cl})\end{array}$ & p-value \\
\hline \multicolumn{4}{|l|}{ Model A } \\
\hline $\begin{array}{l}\text { Histology } \\
\text { Papillary }\end{array}$ & 65 & $0.5 \mathrm{I}(0.27-0.97)$ & $0.040 *$ \\
\hline $\begin{array}{l}\text { CD44s } \\
\text { Positive }\end{array}$ & 47 & $2.13(1.14-3.96)$ & $0.017^{*}$ \\
\hline \multicolumn{4}{|l|}{ Model B } \\
\hline $\begin{array}{l}\text { Histology } \\
\text { Papillary }\end{array}$ & 65 & $0.47(0.25-0.89)$ & $0.021^{*}$ \\
\hline $\begin{array}{l}\text { CD44s and OV IgG } \\
\text { Positive }\end{array}$ & 47 & $2.38(I .17-4.8 I)$ & $0.016 *$ \\
\hline \multicolumn{4}{|l|}{ Model C } \\
\hline $\begin{array}{l}\text { Histology } \\
\text { Papillary }\end{array}$ & 65 & $0.42(0.22-0.80)$ & $0.008^{*}$ \\
\hline $\begin{array}{l}\text { CD44v8-10 and OV IgG } \\
\text { High and Positive }\end{array}$ & 49 & $2.37(1.26-4.45)$ & $0.007 *$ \\
\hline \multicolumn{4}{|l|}{ Model D } \\
\hline $\begin{array}{l}\text { Histology } \\
\text { Papillary }\end{array}$ & 65 & $0.44(0.23-0.85)$ & $0.014^{*}$ \\
\hline $\begin{array}{l}\text { CD44s and CD44v8-I0 } \\
\text { Positive and High }\end{array}$ & 47 & $3.34(17 \mid-6.52)$ & $<0.001 *$ \\
\hline \multicolumn{4}{|l|}{ Model E } \\
\hline $\begin{array}{l}\text { Histology } \\
\text { Papillary }\end{array}$ & 65 & $0.53(0.28-1.03)$ & 0.060 \\
\hline $\begin{array}{l}\text { OV IgG and CD44s and } \\
\text { CD44v8-10 } \\
\text { Positive and Positive and } \\
\text { High }\end{array}$ & 47 & $3.75(1.6 \mathrm{I}-8.72)$ & $0.002 *$ \\
\hline
\end{tabular}

Note: *P value equal to or less than 0.05 was considered statistically significant. Abbreviation: HR, hazard ratio.

reflect the true prevalence of OV associated CCA. Another reason could be the reduction of OV infection incidence. Over the past 30 years there have been many campaigns to eliminate OV infection which have resulted in a decline in the prevalence of OV infection from $89.5 \%$ in $1984^{27}$ to $15.7 \%$ in $2009,{ }^{28}$ and and to $10 \%$ in 2019 from population-based studies. ${ }^{29}$

$\mathrm{OV}$ associated CCA has a grave prognosis as a previous study from Thailand reported 5-year survival after curative resection of only $20 \%,{ }^{17}$ while 5 -year survival after resection from countries with non-OV associated CCA was $30-42 \% .{ }^{18,30}$ A prospective study in 2016 reported that most CCA patients present at an advanced, unresectable stage, $65 \%$ of walk-in patients received only symptomatic care and the resectable cases were only $10 \%{ }^{31}$ In our study more than $60 \%$ of patients who underwent surgery were in stages III or IV and had a poor survival outcome, while in non-OV associated CCA countries resectable cases with stages III or IV only accounted for $6-20 \%$ of the total. ${ }^{19,32,33}$ The aggressiveness of CCA in $\mathrm{OV} \operatorname{IgG}$ positive cases may be explained by immune response inflammatory processes that induce cellular adaptation to oxidative stress, immune evasion and cellular proliferation. This concept is supported by our report of a high level of antibody mediated response resulting in significantly poorer survival.

Because cancer stem cells are believed to be key regulators of tumor aggressiveness, ${ }^{34}$ the levels of cancer stem cell markers may also be used as prognostic markers for patient outcome. The expression of CD44s in CCA from previous studies was $49 \%$ associated with poor survival. ${ }^{14,35}$ Our study showed a similar result with $40 \%$ of cases with CD44s expression having significantly poorer survival in early stage CCA. Moreover, our result also supports a previous study that found a high expression of cancer stem cell markers, CD44s, CD44v6 and CD44v8-10, associated with poor prognosis in early stage CCA patients. ${ }^{36} \mathrm{CD} 44 \mathrm{v} 8-10$ is a variant isoform of CD44 that plays a role in the regulation of oxidative stress in tumors by stabilizing the cystine-glutamate transporter and promoting glutathione synthesis. ${ }^{37}$ In OV induced hamster CCA tissues, the expression of CD44v8-10 was induced during bile duct transformation, and it was associated with a poor prognosis. ${ }^{13}$ In addition, CD44v8-10 showed a higher level in OV associated CCA compared with non-OV associated CCA, and it has been suggested as a marker related to inflammation associated cancer development. ${ }^{38}$ In our result, we found that patients positive for $\mathrm{OV}$ IgG and CD44s with a high expression of CD44v8-10 showed the poorest prognosis compared with other patients in both early and late stages of CCA. This result may be explained by the high level of oxidative stress, which may induce tumor aggressiveness and lead to a poor prognosis.

Survival of CCA patients not only depends on tumor aggressiveness, but also depends on treatment options provided. Surgical resection is the only main treatment which had a chance to achieve long-term survival, 

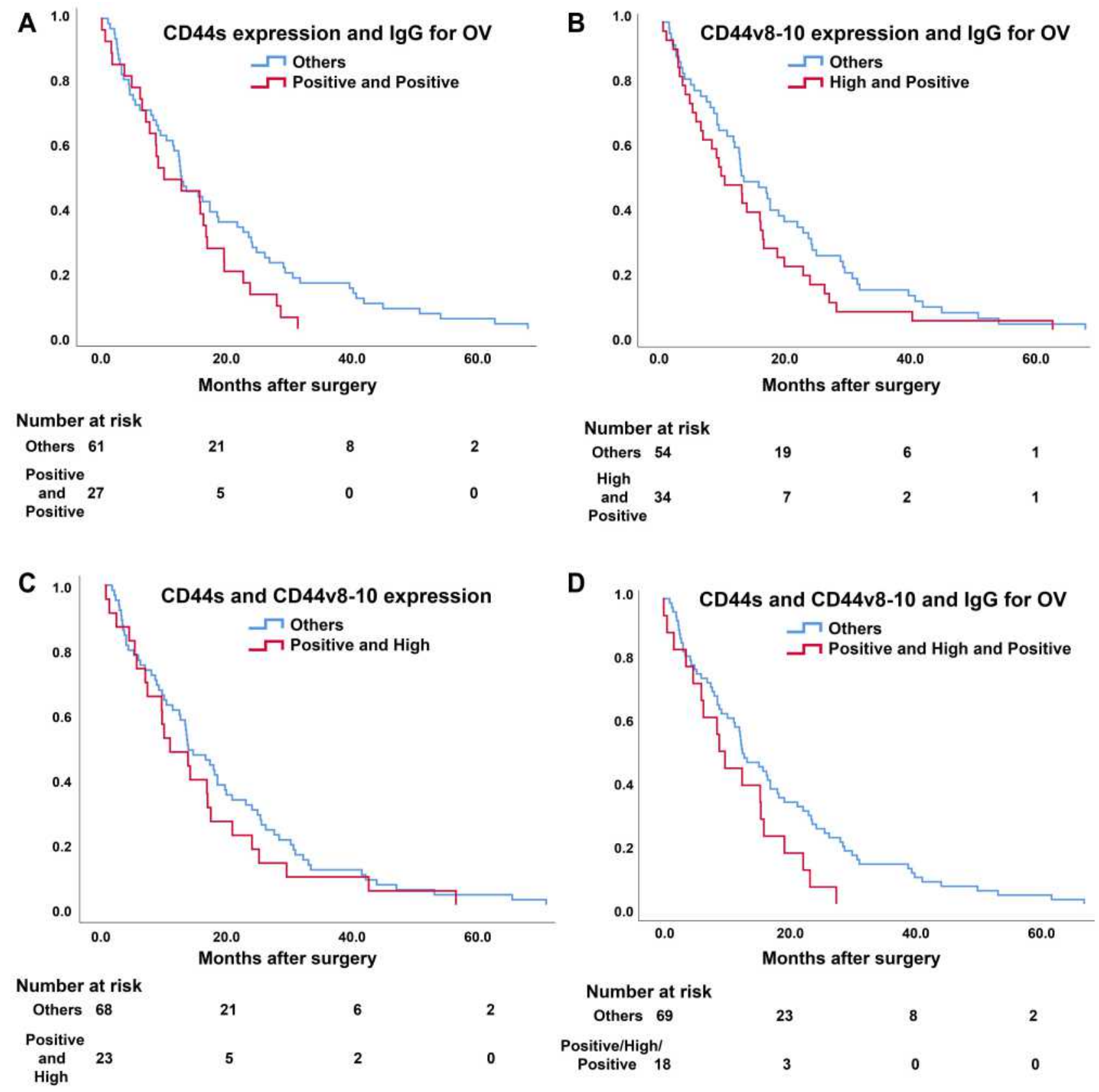

Figure 4 Kaplan-Meier analysis of overall survival with a combination of serum IgG for OV and IHC results in late stage CCA. (A) Survival with a combination of CD44s and IgG for OV. (B) Survival with a combination of CD44v8-10 and IgG for OV. (C) Survival with a combination of CD44s and CD44v8-10. (D) Survival with a combination of CD44s, CD44v8-10 and IgG for OV.

but the 5-year survival rate after resection was unsatisfactory. Adjuvant treatment should be a beneficial option in selected patients by data from a previous retrospective study. ${ }^{39}$ A meta-analysis published in 2019 , that included 30 retrospective studies and 5 randomized trials, reported significantly better overall survival in CCA patients who received any adjuvant chemotherapy compared with surgery alone and reported benefits in the lymph node positive and surgical margin positive groups. ${ }^{40}$ Our study shows the same result, that patients with stages III and IV with adjuvant chemotherapy had better survival. Given this information, we advocate the use of adjuvant chemotherapy for stages III and IV patients. The randomized trial addressed the benefit of adjuvant capecitabine in improving recurrence free survival compared with surgery alone, but overall survival was not different by intention to treat analysis. ${ }^{21}$ However, because of discrepancies of study methods and heterogenicity of patients, the role of 
Table 6 Multivariate Analyses of IHC Results in Late Stage CCA

\begin{tabular}{|l|l|l|l|}
\hline \multicolumn{4}{|c|}{ Late Stage (III, IV) } \\
\hline Variables & N & $\begin{array}{l}\text { Adjusted HR } \\
\text { (95\% CI) }\end{array}$ & p-value \\
\hline $\begin{array}{l}\text { Resection margin } \\
\text { Positive }\end{array}$ & III & $1.54(0.99-2.39)$ & 0.056 \\
\hline $\begin{array}{l}\text { Adjuvant chemotherapy } \\
\text { No }\end{array}$ & $11 \mathrm{I}$ & $1.72(1.08-2.76)$ & $0.023^{*}$ \\
\hline $\begin{array}{l}\text { OV IgG and CD44s and } \\
\text { CD44v8-10 } \\
\text { Positive and Positive and } \\
\text { High }\end{array}$ & 88 & $1.76(1.02-3.03)$ & $0.04 I^{*}$ \\
\hline
\end{tabular}

Note: *P value equal to or less than 0.05 was considered statistically significant. Abbreviation: HR, hazard ratio.

adjuvant treatment remains controversial. The distinctive risk factors and effective biomarkers not only guided prognosis, but also apply for selection of patients for adjuvant treatment in a personalized treatment strategy. ${ }^{22}$ Our study found positive OV IgG and expression of CD44 were significant prognostic factors for poor survival either in early or late stage CCA therefore CD44 pathway targeting is promising for adjuvant treatment. Many advanced preclinical CCA model have recently been rapidly developed, including cell line culture, 3D spheroid model, 3D organoid model and patient's derived xenograft; these model are fundamental tools for initiating personalized treatment. $^{41}$ A recent study reported that sulfasalazine (a CD44 targeting agent) enhanced cytotoxicity of cisplatin in CCA cell line and combined sulfasalazine with cisplatin treatment had significant tumor growth inhibition, increased apoptosis and decreased cell proliferation in high CD44v9 expressed CCA cell implanted xenograft model. $^{42}$ Clinical study of sulfasalazine combined with chemotherapy is promising in $\mathrm{OV}$ associated $\mathrm{CCA}$ or CD44 expressed CCA both in adjuvant and advanced settings.

The limitation of this study was the reliance on retrospective data, therefore some parameters may be imbalanced between groups.

\section{Conclusion}

In summary, OV-associated $\mathrm{CCA}$ is an inflammatory induced cancer with a preventable cause. High levels of antibody mediated immune response to the parasite had consequences for the aggressiveness of CCA. Moreover, a combination of OV IgG level with CD44s and CD44v810 was associated with tumor aggressiveness and showed the poorest prognosis in patients after resection. Therefore, these markers may be used as prognostic markers for CCA patients: targeting and blocking the CD44 pathway is a promising study for adjuvant treatment.

\section{Acknowledgments}

We thank Professor Trevor N. Petney for editing the MS via the Publication Clinic KKU, Thailand.

\section{Funding}

This work was supported by grants from Cholangiocarcinoma Screening and Care Program (CASCAP-13), the National Research Council of Thailand through Fluke Free Thailand Project (FFTT2-

14) and the Basic Research Fund of Khon Kaen University under Cholangiocarcinoma Research Institute allocated to AT.

\section{Disclosure}

The authors report no conflicts of interest in this work.

\section{References}

1. Blechacz B, Gores GJ. Cholangiocarcinoma: advances in pathogenesis, diagnosis, and treatment. Hepatology. 2008;48(1):308-321. doi: $10.1002 /$ hep. 22310

2. Sripa B, Kaewkes S, Sithithaworn P, et al. Liver fluke induces cholangiocarcinoma. PLoS Med. 2007;4(7):e201. doi:10.1371/journal.pmed.0040201

3. Yongvanit P, Pinlaor S, Bartsch H. Oxidative and nitrative DNA damage: key events in opisthorchiasis-induced carcinogenesis. Parasitol Int. 2012;61(1):130-135. doi:10.1016/j.parint.2011.06.011

4. Ito T, Sakurai-Yageta M, Goto A, Pairojkul C, Yongvanit P, Murakami Y. Genomic and transcriptional alterations of cholangiocarcinoma. J Hepatobiliary Pancreat Sci. 2014;21 (6):380-387. doi:10.1002/jhbp.67

5. Titapun A, Techasen A, Sa-Ngiamwibool P, et al. Serum IgG as a marker for opisthorchis viverrini-associated cholangiocarcinoma correlated with HER2 overexpression. Int $J$ Gen Med. 2020;13:1271-1283. doi:10.2147/IJGM.S282519

6. Huang T, Song X, Xu D, et al. Stem cell programs in cancer initiation, progression, and therapy resistance. Theranostics. 2020;10 (19):8721-8743. doi:10.7150/thno.41648

7. Ponta H, Sherman L, Herrlich PA. CD44: from adhesion molecules to signalling regulators. Nat Rev Mol Cell Biol. 2003;4(1):33-45. doi:10.1038/nrm1004

8. Jakob M, Sharaf K, Schirmer M, et al. Role of cancer stem cell markers ALDH1, BCL11B, BMI-1, and CD44 in the prognosis of advanced HNSCC. Strahlenther Onkol. 2021;197(3):231-245. doi:10.1007/s00066-020-01653-5

9. Siddiqui Z, Srivastava AN, Sankhwar SN, et al. Synergic effects of cancer stem cells markers, CD44 and embryonic stem cell transcription factor Nanog, on bladder cancer prognosis. Br J Biomed Sci. 2020;77(2):69-75. doi:10.1080/09674845.2019.1692761 
10. Papadaki C, Manolakou S, Lagoudaki E, et al. Correlation of PKM2 and $\mathrm{CD} 44$ protein expression with poor prognosis in platinum-treated epithelial ovarian cancer: a Retrospective Study. Cancers (Basel). 2020;12(4):1013. doi:10.3390/cancers 12041013

11. Zhou J, Du Y, Lu Y, et al. CD44 expression predicts prognosis of ovarian cancer patients through promoting epithelial-mesenchymal transition (EMT) by regulating snail, ZEB1, and Caveolin-1. Front Oncol. 2019;9:802. doi:10.3389/fonc.2019.00802

12. Wang H, Wang L, Song Y, et al. CD44(+)/CD24(-) phenotype predicts a poor prognosis in triple-negative breast cancer. Oncol Lett. 2017;14(5):5890-5898. doi:10.3892/ol.2017.6959

13. Thanee M, Loilome W, Techasen A, et al. CD44 variant-dependent redox status regulation in liver fluke-associated cholangiocarcinoma: a target for cholangiocarcinoma treatment. Cancer Sci. 2016;107 (7):991-1000. doi:10.1111/cas.12967

14. Morine Y, Imura S, Ikemoto T, Iwahashi S, Saito YU, Shimada M. CD44 expression is a prognostic factor in patients with intrahepatic cholangiocarcinoma after surgical resection. Anticancer Res. 2017;37 (10):5701-5705. doi:10.21873/anticanres. 12007

15. Neradil J, Veselska R. Nestin as a marker of cancer stem cells. Cancer Sci. 2015;106(7):803-811. doi:10.1111/cas.12691

16. Ishiwata T, Matsuda Y, Naito Z. Nestin in gastrointestinal and other cancers: effects on cells and tumor angiogenesis. World J Gastroenterol. 2011;17(4):409-418. doi:10.3748/wjg.v17.i4.409

17. Titapun A, Pugkhem A, Luvira V, et al. Outcome of curative resection for perihilar cholangiocarcinoma in Northeast Thailand. World J Gastrointest Oncol. 2015;7(12):503-512. doi:10.4251/wjgo.v7. i12.503

18. Nagino M, Ebata T, Yokoyama Y, et al. Evolution of surgical treatment for perihilar cholangiocarcinoma: a single-center 34-year review of 574 consecutive resections. Ann Surg. 2013;258 (1):129-140. doi:10.1097/SLA.0b013e3182708b57

19. Hirano S, Kondo S, Tanaka E, et al. Outcome of surgical treatment of hilar cholangiocarcinoma: a special reference to postoperative morbidity and mortality. J Hepatobiliary Pancreat Sci. 2010;17 (4):455-462. doi:10.1007/s00534-009-0208-1

20. DeOliveira ML, Cunningham SC, Cameron JL, et al. Cholangiocarcinoma: thirty-one-year experience with 564 patients at a single institution. Ann Surg. 2007;245(5):755-762. doi:10.1097/01.sla.0000251366.62632.d3

21. Primrose JN, Fox R, Palmer DH, et al. Adjuvant capecitabine for biliary tract cancer: the BILCAP randomized study. J Clin Oncol. 2017;35(15_suppl):4006. doi:10.1200/JCO.2017.35.15_suppl.4006

22. Rizzo A, Brandi G. Pitfalls, challenges, and updates in adjuvant systemic treatment for resected biliary tract cancer. Expert Rev Gastroenterol Hepatol. 2021:1-8.

23. Jariwala AR, Oliveira LM, Diemert DJ, et al. Potency testing for the experimental Na-GST-1 hookworm vaccine. Expert Rev Vaccines. 2010;9(10):1219-1230. doi:10.1586/erv.10.107

24. Schmoor C, Sauerbrei W, Schumacher M. Sample size considerations for the evaluation of prognostic factors in survival analysis. Stat Med 2000;19(4):441-452. 19:4<441::AID-SIM349>3.0.CO;2-N

25. Banales JM, Marin JJG, Lamarca A, et al. Cholangiocarcinoma 2020: the next horizon in mechanisms and management. Nat Rev Gastroenterol Hepatol. 2020;17(9):557-588.

26. Itoh M, Pairojkul C, Thamawit W, et al. Association of antibodies to Opisthorchis viverrini with hepatobiliary disease in northeastern Thailand. Am J Trop Med Hyg. 1994;51(4):424-429. doi:10.4269/ ajtmh.1994.51.424

27. Upatham ES, Viyanant V, Kurathong S, et al. Relationship between prevalence and intensity of Opisthorchis viverrini infection, and clinical symptoms and signs in a rural community in north-east Thailand. Bull World Health Organ. 1984;62(3):451-461.
28. Sithithaworn P, Andrews RH, Van De N, et al. The current status of opisthorchiasis and clonorchiasis in the Mekong Basin. Parasitol Int. 2012;61(1):10-16. doi:10.1016/j.parint.2011.08.014

29. Sornlorm K, Loahasiriwong W, Sithithaworn P, Thinkhamrop W. Influence of geographic, knowledge and behavioral factors on opisthorchis viverrini infection in the Northeast of Thailand. Asian Pac J Trop Med. 2019;12(11):499-506. doi:10.4103/19957645.271289

30. Igami T, Nishio H, Ebata T, et al. Surgical treatment of hilar cholangiocarcinoma in the "new era": the Nagoya University experience. J Hepatobiliary Pancreat Sci. 2010;17(4):449-454. doi:10.1007/ s00534-009-0209-0

31. Luvira V, Nilprapha K, Bhudhisawasdi V, Pugkhem A, Chamadol N, Kamsa-ard S. Cholangiocarcinoma patient outcome in Northeastern Thailand: single-Center Prospective Study. Asian Pac J Cancer Prev. 2016;17(1):401-406. doi:10.7314/APJCP.2016.17.1.401

32. Lee SG, Song GW, Hwang S, et al. Surgical treatment of hilar cholangiocarcinoma in the new era: the Asan experience. J Hepatobiliary Pancreat Sci. 2010;17(4):476-489. doi:10.1007/ s00534-009-0204-5

33. Unno M, Katayose Y, Rikiyama T, et al. Major hepatectomy for perihilar cholangiocarcinoma. J Hepatobiliary Pancreat Sci. 2010;17 (4):463-469. doi:10.1007/s00534-009-0206-3

34. Ayob AZ, Ramasamy TS. Cancer stem cells as key drivers of tumour progression. J Biomed Sci. 2018;25(1):20. doi:10.1186/s12929-0180426-4

35. Ashida K, Terada T, Kitamura Y, Kaibara N. Expression of E-cadherin, alpha-catenin, beta-catenin, and CD44 (standard and variant isoforms) in human cholangiocarcinoma: an immunohistochemical study. Hepatology. 1998;27(4):974-982. doi:10.1002/ hep. 510270412

36. Padthaisong S, Thanee M, Namwat N, et al. Overexpression of a panel of cancer stem cell markers enhances the predictive capability of the progression and recurrence in the early stage cholangiocarcinoma. J Transl Med. 2020;18(1):64. doi:10.1186/ s12967-020-02243-w

37. Ishimoto T, Nagano O, Yae T, et al. CD44 variant regulates redox status in cancer cells by stabilizing the XCT subunit of system xc(-) and thereby promotes tumor growth. Cancer Cell. 2011;19 (3):387-400. doi:10.1016/j.ccr.2011.01.038

38. Suwannakul N, Ma N, Thanan R, et al. Overexpression of CD44 variant 9: a novel cancer stem cell marker in human cholangiocarcinoma in relation to inflammation. Mediators Inflamm. 2018;2018:4867234. doi:10.1155/2018/4867234

39. Wirasorn K, Ngamprasertchai T, Khuntikeo N, et al. Adjuvant chemotherapy in resectable cholangiocarcinoma patients. J Gastroenterol Hepatol. 2013;28(12):1885-1891. doi:10.1111/jgh.12321

40. Rangarajan K, Simmons G, Manas D, Malik H, Hamady ZZ. Systemic adjuvant chemotherapy for cholangiocarcinoma surgery: a systematic review and meta-analysis. Eur J Surg Oncol. 2020;46 (4 Pt A):684-693. doi:10.1016/j.ejso.2019.11.499

41. Massa A, Varamo C, Vita F, et al. Evolution of the experimental models of cholangiocarcinoma. Cancers. 2020;12(8):2308. doi:10.3390/cancers 12082308

42. Thanee M, Padthaisong S, Suksawat M, et al. Sulfasalazine modifies metabolic profiles and enhances cisplatin chemosensitivity on cholangiocarcinoma cells in in vitro and in vivo models. Cancer Metab. 2021;9(1):11. doi:10.1186/s40170-021-00249-6 


\section{Publish your work in this journal}

The International Journal of General Medicine is an international, peer-reviewed open-access journal that focuses on general and internal medicine, pathogenesis, epidemiology, diagnosis, monitoring and treatment protocols. The journal is characterized by the rapid reporting of reviews, original research and clinical studies across all disease areas. The manuscript management system is completely online and includes a very quick and fair peer-review system, which is all easy to use. Visit http://www.dovepress.com/ testimonials.php to read real quotes from published authors. 